IAU Colloquium 164: Radio Emission from Galactic and Extragalactic Compact Sources

ASP Conference Series, Vol. 144, 1998

J. A. Zensus, G. B. Taylor, \& J. M. Wrobel (eds.)

\title{
Giga-bit VLBI Data Recorder, New Concept of Formatter Independent
}

\author{
J. Nakajima, Y. Koyama, M. Sekido, \\ H. Kiuchi, S. Hama, T. Kondo, \& Y. Takahashi \\ Communications Research Laboratory, 893 Hirai Kashima Ibaraki 314 Japan
}

N. Kawaguchi, Y. Chikada, \& M. Miyoshi

National Astronomical Observatory, 2-21-1 Osawa Mitaka Tokyo 181 Japan

\begin{abstract}
A high performance VLBI recorder project using advanced digital technology started in 1995. TOSHIBA GBR1000 and VLBI interface define 32 parallel, $32 \mathrm{MHz}$ clock standard digital interface and support transparent recording/play-back to extremely high-speed digital bit stream. This is the formatter independent scientific recording bind the UTC to observed data. This highly reliable, low-bit-error-rate $\left(10^{-16}\right)$ recording system is also expected to be used in other scientific field. For the first example to examine the recorder performance in VLBI, we had been prepared 1024 Mbps sampler (256/512/1024 MSps, 2 bit, 4/2/1 ch). And here, an experimental giga-bit correlator specification for these VLBI acquisition system is introduced.
\end{abstract}

\section{Giga-bit Recorder}

For the wide-band recording, we had examined cassette digital recorders since 1994 and we chose the GBR-1000 (Endo et al. 1994). This VCR is designed for high-definition TV (HDTV) spec digital video without compression. Data compression VCRs can not be used for 1-bit noise recording. The GBR raw data rate on metal-particle magnetic tape is nearly $1200 \mathrm{Mbps}$. The $1024 \mathrm{Mbps}$ user data is protected by Reed-Solomon product code in rest. Commands to the recorder are handled by a DRA1000 interface (by Toshiba \& YEM) by GPIB protocols (Nakajima et al. 1997). These command are issued from PC/FS9 at observation site. The interface maintains the UTC and convert observed time to corresponding HDTV flame of 30-times in a second. The recorder has several advanced functions no former VLBI recorder provided. For example, display self diagnose of 16 heads from read-after-write, error history logging of tape, and identification of recording station without tape label. Upto 8-units can be synchronized without extra hardware. The physical size of the cassette SMPTE defined D-6, $3 / 4$ inch tape inside, is same as that of K4 cassette (Noda et al. 1994). The GBR consist of separable transport unit and digital processor. They are portable as well as former K4 VLBI system. For your recording application, you can obtain information of the GBR recorder at http://www.crl.go.jp/ka/radioastro.

\section{Giga-bit Correlator}

The Giga-bit correlator is a simple experimental version. Used Correlation core LSIs are UWBC (Ultra WideBand Correlator) chips already proved its performance at the Nobeyama Millimeter Array. OKI Electric is integrating the onebaseline correlator named UWBC-GICO (GIga-bit COrrelator). The correlator architecture is rather simple. Because we do not need time-code related hard- 
ware. For global VLBI delay tracking, we will make additional delay tracker. It is especially noteworthy that the correlator of connected interferometer is used for VLBI. The correlation mode is aimed just for $1024 \mathrm{Mbps}, 1$ bit. The 1024 Mbps sampler is connected to the GICO correlator directly through the $32 \mathrm{MHz}$ $\mathrm{x} 32$ parallel interface and the system is under auto-correlation experiment.

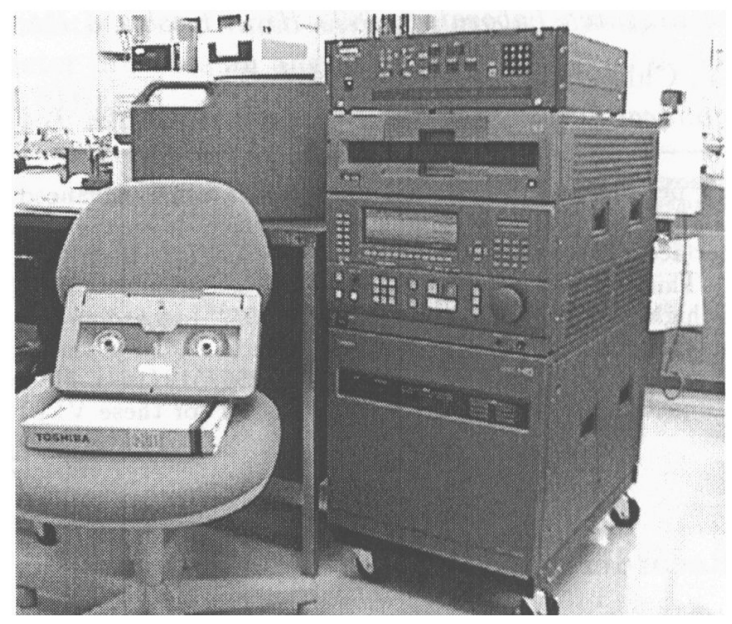

Figure 1. GBR-1000 transport and processor under experiment. Upper small unit is the DRA-1000 interface. Cassette size of the D-6 is same to the former K4(ID-1). The Data packing density is 4 times higher. components.

Table 1. Comparison of the GBR and K4-VSOP VLBI

\begin{tabular}{lcc}
\hline & GBR \& GICO & K4-VSOP \& FX \\
\hline Recording Speed(1) & $1024 \mathrm{Mbps}$ & $128 \mathrm{Mbps}$ \\
Recording format & $\mathrm{D} 6$ & $\mathrm{I}-\mathrm{D} 1$ \\
Channel configuration(2) & $512 \mathrm{MHz} 1 \mathrm{bit} 1 \mathrm{ch}$ & $32 \mathrm{MHz} 2 \mathrm{bit} 2 \mathrm{ch}$ \\
Rec. duration / tape & $60 \mathrm{~min}$ & $240 \mathrm{~min}$ \\
Time code & none & on/off \\
Correlator type & XF & $\mathrm{FX}$ \\
Fringe tracking & Global & Space \\
\hline
\end{tabular}

Notes: 1: K4 recorder is max 256 Mbps. 2: Typical channel configurations.

\section{References}

Endo, N., et al. 1994. in IEEE Denshi Tokyo, No. 33, 236-239.

Noda, C., et al. 1994. in Proc, of 1994 European SMPTE Conference.

Nakajima, J., et al. 1997. in Proc. of International Symposium on High Sensitivity Radio Astronomy eds. N. Jackson \& R.J. Davis (Cambridge: Cambridge Univ. Press), 256259.

Nakajima, J., et al. 1997. in Proc. of Technical Workshop, APT and APSG, CRL, 171-175. 